\title{
Local conformational fluctuations can modulate the coupling between proton binding and global structural transitions in proteins
}

\begin{abstract}
Steven T. Whitten* ${ }^{\dagger}$, Bertrand García-Moreno E. ${ }^{\ddagger}$, and Vincent J. Hilser*§ October 8, 2004)

Local conformational fluctuations in proteins can affect the coupling between ligand binding and global structural transitions. This finding was established by monitoring quantitatively how the population distribution in the ensemble of microstates of staphylococcal nuclease was affected by proton binding. Analysis of acid unfolding and proton-binding data with an ensemble-based model suggests that local fluctuations: $(i)$ can be effective modulators of ligand-binding affinities, (ii) are important determinants of the cooperativity of ligand-driven global structural transitions, and (iii) are well represented thermodynamically as local unfolding processes. These studies illustrate how an ensemble-based description of proteins can be used to describe quantitatively the interdependence of local conformational fluctuations, ligand-binding processes, and global structural transitions. This level of understanding of the relationship between conformation, energy, and dynamics is required for a detailed mechanistic understanding of allostery, cooperativity, and other complex functional and regulatory properties of macromolecules.
\end{abstract}

* Department of Human Biological Chemistry and Genetics, University of Texas Medical Branch, Galveston, TX 77555; ${ }^{\dagger}$ RedStorm Scientific, Galveston, TX 77550; and ₹Department of Biophysics, The Johns Hopkins University, Baltimore, MD 21218

Edited by William F. DeGrado, University of Pennsylvania School of Medicine, Philadelphia, PA, and approved February 9,2005 (received for review

cooperativity | ensemble | linkage | dynamics | electrostatics

$\mathbf{T}$ he native states of proteins are conformationally heterogeneous (1-8). This characteristic is primarily the result of local structural fluctuations that are thought to contribute to stability and to function (9-11). As a consequence of these spontaneous conformational fluctuations, proteins in solution exist as ensembles of closely related, transient, and interconverting conformational microstates that, when averaged, describe the conformational state represented by the crystal structure. NMR spectroscopy can be used to study the very fast motions of proteins $(9-11)$. Nevertheless, the probability and structural character of the full spectrum of microstates sampled by proteins is not yet known $(12,13)$. The sensitivity of the ensemble of microstates to changes in environmental conditions (i.e., $\mathrm{pH}$, temperature, pressure, ligand binding, and concentrations of osmolytes and denaturants) is also not well understood, and neither is the manner in which local fluctuations are coupled to larger, more global structural transitions. Here, we introduce an ensemble-based description of proteins that accounts for the complex interplay between local conformational fluctuations, global stability, and ligand binding. This ensemble view of proteins can contribute innovative mechanistic insight into the evolution of enzymatic and regulatory functions of proteins.

To investigate the coupling between ligand binding, conformational fluctuations, and global stability, the proton-binding properties of staphylococcal nuclease (SNase) were studied. Proton-binding processes are particularly useful for this purpose because they are experimentally measurable and because the $\mathrm{pK}_{\mathrm{a}}$ values of ionizable groups are exquisitely sensitive to their local microenvironments (14-27). Each proton-binding site acts as a site-specific probe of local conformation.

Proton-binding processes are also of special interest because many proteins have evolved to harness small differences in $\mathrm{pH}$ to trigger physiologically important conformational changes. The regulation of the oxygen affinity by hemoglobin (28), the activation of bacterial toxins (29), and key steps in the infectivity cycles of viruses as diverse as polio (30), influenza (31-33), and HIV (34) are examples of physiologically relevant processes in which function is regulated through proton-linked structural transitions. Understanding the relationship between local conformational fluctuations, ligand (i.e., $\mathrm{H}^{+}$) binding, and global structural transformations in these proteins is necessary to elucidate the physical basis of their function.

Molecular dynamics (MD) simulations and other all-atom approaches have been useful for describing the character and consequences of local conformational fluctuations (35-39), as well as providing significant functional insight (40). However, in the relatively short time scales that can be accessed routinely by MD methods, local backbone relaxations, and other slow relaxation events likely to be relevant to biological and equilibrium thermodynamic processes $(12,13)$ are not sampled sufficiently. The alternative approach that is described in this work focuses on the quantitative description of the instantaneous ensemble constituted by the different microstates populated by the native states of proteins in solution (41). If the structures of the different microstates can be sampled adequately, and a reliable energy function can be used to calculate the energy of each microstate, then a Boltzmann-weighted scheme (41) can be used to calculate global solution properties of the protein as a sum of contributions by individual microstates. Here, we show how this simple ensemble-based approach can be used to describe complex processes, such as the acid-driven unfolding of a protein. The ensemble-based approach enables a detailed description of the $\mathrm{pH}$-driven shift in the character of the instantaneous ensemble wherein the coupling between ligand binding, local conformational fluctuations, and global structural transitions is described explicitly.

\section{Methods}

Generation of the Ensemble. The crystal structure of SNase (Protein Data Bank ID code 1STN; ref. 42) was used as a template to generate a model of the ensemble by using the COREX algorithm described in refs. 41 and 43-46. In the COREX calculations, the structure for each microstate is generated by treating local fluctuations as local folding-unfolding reactions that occur in an otherwise folded and native-like protein (Fig. 1A). In the present study, $>10^{6}$ different conformational microstates were generated by systematically varying the number and location of fluctuating (locally unfolded) residues. The probability of each

This paper was submitted directly (Track II) to the PNAS office.

Abbreviations: FDPB, Finite Difference Poisson-Boltzmann; GCP, global coupling parameters; MD, molecular dynamics; SNase, staphylococcal nuclease.

§To whom correspondence should be addressed. E-mail: vjhilser@utmb.edu.

C 2005 by The National Academy of Sciences of the USA 
A

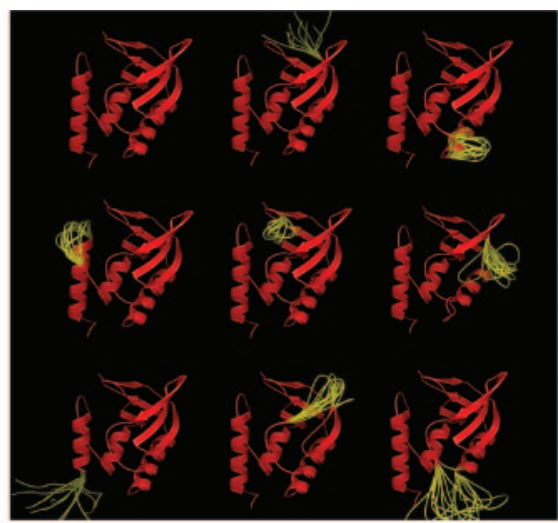

B

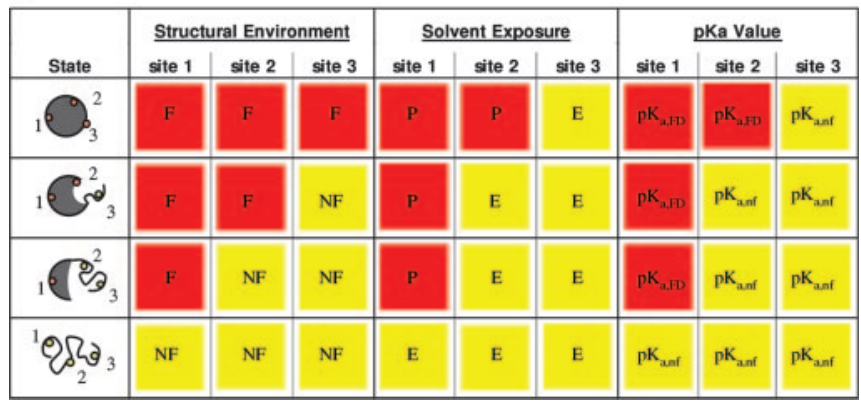

Fig. 1. Schematic representation of the SNase ensemble calculated for the COREX/BEST algorithm. $(A)$ Nine of the most probable conformational states (of $\approx 10^{6}$ ) of the SNase ensemble, modeled as described in ref. 47. Each microstate exhibits dual structural character and consists of a mixture of regions that are considered to be native-like (red) and regions that are treated thermodynamically as unfolded (yellow). Molecular diagrams in this figure were made by using the program PYMOL (65). (B) Ensemble model used to describe quantitatively the coupling between local unfolding energetics and the energetics of proton binding. Depicted are the proton titration properties of a hypothetical protein (gray circle) that has only three titratable groups (dots) and whose conformational ensemble contains only four microstates: a fully folded, two partially folded, and a fully unfolded state. Unfolded segments of the protein are depicted as randomly curved lines. The small dots represent the individual titratable groups of the protein: sites 1, 2, and 3. Each individual protonbinding site can reside in a folded (F) or an unfolded (NF) region, and they can be exposed to solvent $(E)$ or protected from solvent $(P)$. The $\mathrm{pK}_{\mathrm{a}}$ value of each titratable group in each state was assigned according to the degree of solvent exposure of the titratable atoms. Groups that were protected from solvent were assigned the $\mathrm{pK}_{\mathrm{a}}$ values calculated with Poisson-Boltzmann continuum electrostatics method $\left(\mathrm{pK}_{\mathrm{a}, \mathrm{FD}}\right)$. Groups that were exposed to solvent were assigned $\mathrm{pK}_{\mathrm{a}}$ values identical to those observed in unfolded polypeptides (pK $a, n f)$.

microstate $\left(P_{i}\right)$ was estimated by using a structure-based calculation with a parameterized energy function that has been calibrated previously and tested extensively (47-55). This probability can be described by the Boltzmann relationship,

$$
P_{i}=\frac{K_{i}}{\sum_{i} K_{i}},
$$

where the statistical weight of each state $\left(K_{i}\right)$ is determined by the relative Gibbs free energy of that state $\left(K_{i}=e^{-\Delta G i / R T}\right.$, where $R$ is the gas constant and $T$ is absolute temperature), and the summation is over all states in the ensemble.

pH Dependence of the Ensemble. To account for proton effects on the probability of each state generated by COREX, we introduce the BEST (Biology using Ensemble-based Structural Thermody- namics) algorithm. In this algorithm, the statistical formula to calculate probability (Eq. 1) was expanded to account for proton-binding energies,

$$
P(p H)_{i}=\frac{K_{i} \cdot \prod_{j}\left(1+10^{\left(p K_{\mathrm{a}, i, j}-p H\right)}\right)}{\sum_{i}\left(K_{i} \cdot \prod_{j}\left(1+10^{\left(p K_{\mathrm{a}, i, j}-p H\right)}\right)\right)},
$$

where $\mathrm{pK}_{\mathrm{a}, i, j}$ is the $\mathrm{pK}_{\mathrm{a}}$ value of residue $j$ in microstate $i$. For each microstate of the ensemble, each ionizable group can have one of two $\mathrm{pK}_{\mathrm{a}}$ values: the $\mathrm{pK}_{\mathrm{a}}$ value in the native conformation $\left(\mathrm{pK}_{\mathrm{a}, \mathrm{FD}}\right)$ or that of the unfolded state $\left(\mathrm{pK}_{\mathrm{a}, \mathrm{nf}}\right)$. For this reason, the distribution of microstates is $\mathrm{pH}$-dependent. The set of rules summarized in Fig. $1 B$ was used to assign $\mathrm{pK}_{\mathrm{a}}$ values to each ionizable group in each of the different microstates of the ensemble. The set of $\mathrm{pK}_{\mathrm{a}, \mathrm{nf}}$ values used are those of model compounds in water $(56,57)$. The $\mathrm{pK}_{\mathrm{a}, \mathrm{FD}}$ values that were used were calculated by using the native-state structure and continuum electrostatic methods based on the numerical solution of the linearized Poisson-Boltzmann equation with the method of finite differences (58). The ionizable groups in the $\mathrm{N}$ - and $\mathrm{C}$-terminal regions of the protein, which are not resolved in any known crystal structure of $\mathrm{SN}$, were assigned $\mathrm{pK}_{\mathrm{a}, \mathrm{nf}}$ values.

Individual groups were assigned $\mathrm{pK}_{\mathrm{a}}$ values based on the criteria of solvent accessibility. When the averaged solvent accessibility of the ionizable atoms of a titratable group was $<31 \%$ (for Glu, Asp, Tyr, Lys, and Arg) or $45 \%$ (for His), these groups were assigned $\mathrm{pK}_{\mathrm{a}, \mathrm{FD}}$ values; all other groups were assigned $\mathrm{pK}_{\mathrm{a}, \mathrm{nf}}$ values. This cutoff maximized the agreement between the calculated and measured overall proton-binding curve of SNase (discussed below). Use of such a seemingly arbitrary cutoff was validated by the ability of the ensemble calculations thus performed to correctly identify the subset of ionizable groups that were shown experimentally to govern the acid unfolding reaction.

Proton Titration Properties of the Ensemble. In an ensemble-based calculation, any measurable solution property of a protein can be calculated as the probability-weighted sum of the individual contribution of all conformational states present in solution under a given set of conditions. In the case of proton titrations, the average degree of protonation at each $\mathrm{pH},\langle Z\rangle$, is calculated as the sum of the titrated protons, $Z_{i}$, in each microscopic state, $\langle Z\rangle=\sum_{i}\left(\mathrm{Z}_{i} \cdot P_{i}\right)$, where $P_{i}$ is the $\mathrm{pH}$-dependent probability of state $i$ calculated with Eq. 2 .

\section{Results and Discussion}

Modulation of the Ensemble by pH. To determine how the character of the ensemble of a protein is affected by changes in $\mathrm{pH}$, the distribution of microstates in the ensemble of SNase was determined at different $\mathrm{pH}$ values. The effect of $\mathrm{pH}$ on the probability of the different microstates arises from the assignment of either $\mathrm{pK}_{\mathrm{a}, \mathrm{nf}}$ or $\mathrm{pK}_{\mathrm{a}, \mathrm{FD}}$ values to each ionizable group in the different microstates. This effect, in turn, depends on whether the titratable atom exists in a native-like or unfolded microenvironment.

The $\mathrm{pH}$ sensitivity of the ensemble of microstates for SNase is shown in Fig. 2, where the free energies of the $\approx 10^{6} \mathrm{mi}-$ crostates are shown at three different $\mathrm{pH}$ values. Under conditions of low $\mathrm{pH}$, the microstates with a higher affinity for protons (those with titratable groups with more normal $\mathrm{pK}_{\mathrm{a}}$ values) are stabilized preferentially over the microstates with lower protonbinding affinity. However, at $\mathrm{pH} 7$ the most probable (i.e., lowest energy) microstates in the ensemble are mostly native, with some locally unfolded sections (i.e., high degrees of fraction native; see Fig. 2). Interestingly, at $\mathrm{pH} 7$ the SNase ensemble is populated mainly by microstates that have at least some fraction of the 

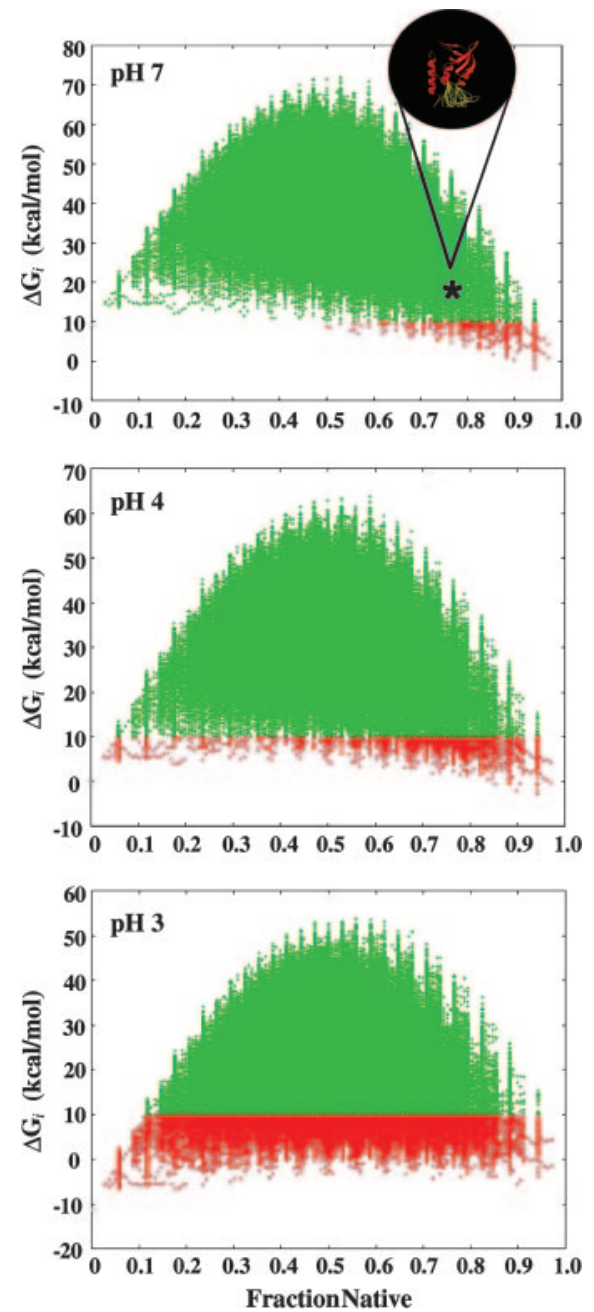

Fig. 2. Relative stability (Gibbs free energy, $\Delta G$ ) of each of the $\approx 10^{6}$ microstates plotted as a function of the fraction native (number of residues in folded segments/total number of residues) at three different $\mathrm{pH}$ values $(\mathrm{pH} 7$, 4 , and 3). Each point corresponds to a particular microstate, and the $\Delta G$ values were calculated relative the fully folded state (i.e., the stability of the fully folded state in the ensemble is 0 at all $\mathrm{pH}$ values). To highlight the changing character of the ensemble induced by $\mathrm{pH}$, all states with $\Delta G$ of $<10 \mathrm{kcal} / \mathrm{mol}$ are shown in red, and those states with $\Delta G$ of $>10 \mathrm{kcal} / \mathrm{mol}$ are shown in green.

molecule unfolded (Figs. 2 and $3 A$ ). Although the stability of each of these partially unfolded states is predicted to be comparable to the stability of the fully folded state, the aggregate probability of these partially unfolded states actually dominates the conformational ensemble at neutral $\mathrm{pH}$.

The $\mathrm{pH}$ sensitivity of the conformational character of the ensemble is of particular significance. States with a lower fraction native become favored as $\mathrm{pH}$ is decreased (Figs. 2 and $3 A$ ). However, this shift does not occur in a gradual, noncooperative fashion. Instead, as the $\mathrm{pH}$ is lowered from 7.0 to 4.0 , the ensemble transforms in a highly cooperative fashion from one that is dominated by native-like microstates into one consisting of microstates that are largely unfolded (Fig. $3 A$ ). Under no conditions of $\mathrm{pH}$ are states with intermediate values of fraction native (i.e., $0.2<x<0.8$ ) populated to a significant extent. Inspection of Fig. $3 B$ reveals that the cooperativity (i.e., the steepness of the acid unfolding transition, which is related to the number of protons bound preferentially by the acid unfolded state relative to the native state) and the midpoint of the acid
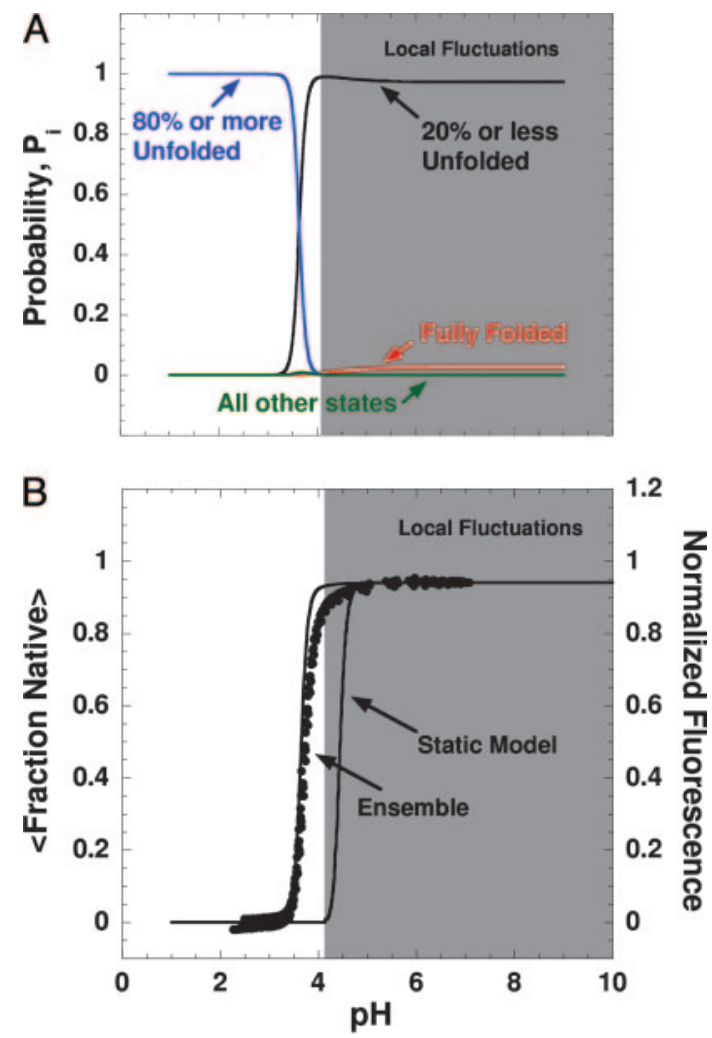

Fig. 3. $\mathrm{pH}$-induced modulation of the SNase ensemble. $(A)$ Calculated $\mathrm{pH}$ dependence of the summed probability of all partially folded states in which $20 \%$ or fewer of the residues are unfolded (black), the summed probability of all states in which $80 \%$ or more of the residues are unfolded (blue), the probability of the fully folded state (red), and the summed probability of all other states of the ensemble (green). (B) Experimental acid unfolding of wild-type (WT) SNase monitored by $\mathrm{pH}$ titration of the intrinsic fluorescence of Trp-140 (24). The lines overlaid on the experimental data represent the ensemble-calculated $\mathrm{pH}$-dependent unfolding of the protein. The relative extent of folding of the ensemble was calculated by $\langle$ Fraction Native $\rangle=\Sigma_{i}$ Fraction Native $i \cdot P(\mathrm{pH})_{i}$. Also shown is the predicted acid unfolding transition based on $\mathrm{pK}_{\mathrm{a}}$ values calculated with Poisson-Boltzmann electrostatics (58). Regions shaded gray in $A$ and $B$ indicate conditions wherein the conformational excursions are dominated by local fluctuations.

unfolding transition monitored experimentally by intrinsic fluorescence are reproduced quantitatively by the ensemble-based calculations.

Because the physical properties and the energy of each state in the ensemble is determined from a consistent set of principles (Fig. $1 B$ and ref. 41), the predicted cooperativity is not an artifact imposed by a bias in the ensemble calculations. Instead, it indicates that, although the individual microscopic states and the associated proton-binding properties calculated with the ensemble method use simple principles, they nonetheless provide an accurate estimate of the average physical character as well as the average proton-binding properties of the dominant states in the native-state ensemble.

Coupling of Proton Binding to Local Fluctuations. The agreement between the proton-binding behavior calculated with the ensemble-based method and the behavior measured experimentally (Fig. 4) is consistent with a significant coupling between proton-binding reactions and local fluctuations. Note in Fig. $4 A$ that in the neutral range of $\mathrm{pH}$, the ensemble-averaged protonbinding properties of SNase resemble the binding properties of the fully folded state. At low $\mathrm{pH}$, the ensemble-calculated proton titration properties correspond to those of a fully unfolded state. 

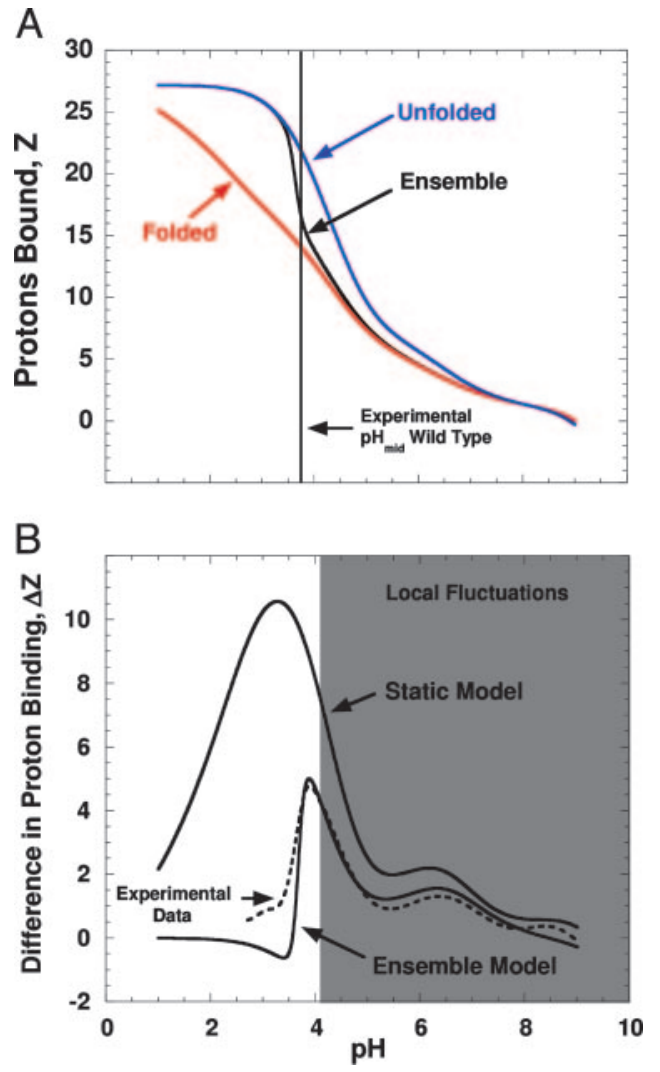

Fig. 4. Proton-binding properties of the SNase ensemble. (A) Calculated proton titration of the ensemble (black lines), the fully native state (red), and the fully unfolded state (blue) for SNase (solid lines). The proton-binding curves were all zeroed at $\mathrm{pH} 9.0$ to facilitate comparison of the different curves. The vertical line describes the midpoint of the $\mathrm{pH}$-induced transition measured experimentally (Fig. 3B). (B) Experimental and calculated preferential proton binding of the fully unfolded state relative to the fully native state and to the ensemble of SNase. The difference in proton binding between $\mathrm{GdnHCl}$ unfolded protein (measured in $6 \mathrm{M} \mathrm{GdnHCl} / 100 \mathrm{mM} \mathrm{KCl}$ at $20^{\circ} \mathrm{C}$ ) and native protein $\left(100 \mathrm{mM} \mathrm{KCl}\right.$ at $20^{\circ} \mathrm{C}$ ) is shown by the dashed lines (24). The curve marked "Static Model" reflects the difference between proton binding of the fully unfolded state achieved in $6 \mathrm{M} \mathrm{GdnHCl}$ and the fully native state, calculated with the $\mathrm{pK}_{\mathrm{a}}$ values obtained with continuum electrostatic methods (FDPB) applied to the $x$-ray structure (Protein Data Bank ID code 1STN). The curve marked "Ensemble Model" represents the difference in proton binding between the fully unfolded state and the ensemble. Gray shading is as described in Fig. 3.

At intermediate $\mathrm{pH}$ values, however, the calculated proton titration curve of the $\mathrm{pH}$-modulated ensemble is between the titration curves of the fully folded and the fully unfolded states (Fig. $4 A$ ). The proton titration properties of the $\mathrm{pH}$-modulated ensemble monitor the same proton-linked global structural transition that is monitored experimentally (Fig. 3B). To appreciate the significance of the agreement between the experimentally determined number of protons bound upon acid unfolding and the value calculated with the ensemble-based approach (Fig. $4 B)$, note that in contrast, the proton uptake upon acid unfolding that was calculated by using the $\mathrm{pK}_{\mathrm{a}}$ values obtained from Finite Difference Poisson-Boltzmann (FDPB) methods applied to only the static crystal structure of the fully native state (58) significantly overestimates the number of protons that are bound upon acid unfolding (Fig. 4B), as well as the midpoint of the $\mathrm{pH}$ induced structural transition (Fig. $3 B$ ).

Microscopic Origins of $\mathbf{p H}$ Sensitivity. According to the structurebased $\mathrm{pK}_{\mathrm{a}}$ calculations with FDPB methods, 16 of the 17 structurally resolved carboxylic residues in SNase titrate with depressed $\mathrm{pK}_{\mathrm{a}}$ values, owing to the existence of a large number of relatively weak, medium- to long-range Coulomb interactions between carboxylic residues and the many basic residues on the surface of SNase (C. A. Fitch, S.T.W., V.J.H., and B.G.-M.E., unpublished results). We note that this behavior is not a subtle feature of the FDPB calculation [i.e., even in calculations where the protein is treated artificially with the high dielectric constant of 80 for water, the $\mathrm{pK}_{\mathrm{a}}$ values of nearly all Glu and Asp residues are depressed to some degree (C. A. Fitch, S. T. Whitten, and B.G.-M.E., unpublished results)]. Clearly, such a result is difficult to reconcile with the experimental proton-binding experiments (Fig. $4 B$ ), which indicate that a subset of the carboxylic residues must titrate with more normal $\mathrm{pK}_{\mathrm{a}}$ values. The ensemble approach described here provides a means of explicitly considering the impact of conformational fluctuations on the titration behavior, and the resulting picture of the ensemble can be experimentally validated on a site-specific basis by determining the coupling between the titration of each residue and the global structural transition, as shown below.

According to the ensemble calculations, a subset of Glu and Asp groups titrates with normal $\mathrm{pK}_{\mathrm{a}}$ values because they exist in regions of the protein that are predicted to be inherently unstable and therefore to be unfolded in many of the most probable microstates in the ensemble. A detailed inspection of the $\mathrm{pH}$-dependent distribution of the conformational ensemble shows that 7 of the 17 carboxylic residues (Fig. $5 A$ ) titrate with normal $\mathrm{pK}_{\mathrm{a}}$ values (i.e., above the midpoint of the acid unfolding transition). In the ensemble calculations, the titration of these seven groups is not coupled thermodynamically to the global structural transition; thus, the calculations reproduce the experimental proton uptake upon acid unfolding (Fig. $4 B$ ). Note that for the case of acid unfolding at a $\mathrm{pH}$ value close to the normal $\mathrm{pH}$ where carboxylic residues titrate, the results of the ensemble calculations are fully independent of the set of $\mathrm{pK}_{\mathrm{a}, \mathrm{FD}}$ values used, provided that they are depressed relative to the set of $\mathrm{pK}_{\mathrm{a}, \mathrm{nf}}$ values.

To assess the validity of the predictions obtained with the ensemble-based calculations, it was necessary to identify experimentally the 10 residues that are predicted by the ensemble calculations to titrate concomitant with the global unfolding transition. Because SNase unfolds in the $\mathrm{pH}$ range where carboxylic residues normally titrate, it was not possible to identify these groups by monitoring their $\mathrm{pK}_{\mathrm{a}}$ shifts with NMR spectroscopy. Instead, these groups were identified by measurement of the global unfolding properties of variants with single Glu $\rightarrow$ Ala and Asp $\rightarrow$ Ala substitutions. If local fluctuations allow a proton to be bound at $\mathrm{pH}$ values above the range of global unfolding (i.e., the $\mathrm{pK}_{\mathrm{a}}$ value in the native ensemble becomes normal), then the substitution with Ala should not affect the global unfolding profile. Conversely, the unfolding profile should be affected when a group whose titration is coupled to the global unfolding is substituted with Ala. The results of the mutational experiments shown in Fig. $5 B$ indicate that the subset of residues identified by the ensemble-based calculations as the residues that bind protons concomitant with unfolding (Fig. $5 A$ ) are indeed the sites where the Ala substitutions were found experimentally to affect the acid unfolding behavior. The ability of the ensemblebased calculation to accurately model the macroscopic proton uptake and acid unfolding transition, as well as to correctly identify those titratable residues that are responsible for the macroscopic behavior, validates the protocol used for the ensemble calculations, which includes the energy function, the representation of the microstates in the ensemble, and the seemingly arbitrary cutoff criteria used to assign $\mathrm{pK}_{\mathrm{a}}$ values. The results also illustrate an important role of local fluctua- 


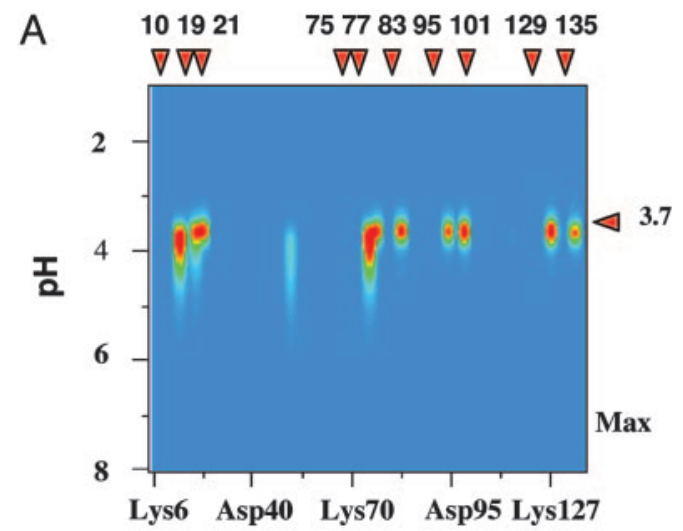

Titratable Residues
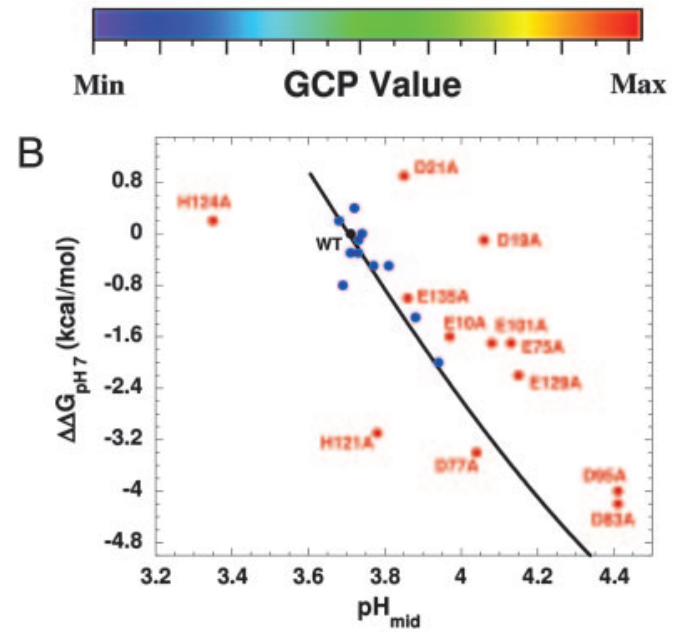

Fig. 5. Microscopic origins of the $\mathrm{pH}$-dependent stability of SNase. (A) Global coupling parameters (GCP) were used to identify coupling between proton binding and global conformational stability: $\mathrm{GCP}(\mathrm{pH})_{j}=\left|\mathrm{Z}(\mathrm{pH})_{f, j}-\left\langle\mathrm{Z}(\mathrm{pH})_{j}\right\rangle\right| \times$ $\left|Z(\mathrm{pH})_{n f, j}-\left\langle Z(\mathrm{pH})_{j}\right\rangle\right|$. The values $Z(\mathrm{pH})_{f, j}$ and $Z(\mathrm{pH})_{n f, j}$ are the $\mathrm{pH}$-dependent protonation states of residue $j$ in the fully folded and fully unfolded states, respectively. $\left\langle Z(\mathrm{pH})_{j}\right\rangle$ is the ensemble-weighted value, equal to $\Sigma_{i}$ $Z(\mathrm{pH})_{i, j} \cdot P(\mathrm{pH})_{i}$. GCP of $>0$ identifies residues whose titration is affected by the $\mathrm{pH}$-dependent redistribution of the conformational ensemble (i.e., their titration is coupled to the acid unfolding). GCP $=0$ identifies residues whose titration is not affected by the $\mathrm{pH}$-induced redistribution of the ensemble. The high GCP values are concentrated near $\mathrm{pH}_{\text {mid }}$ because they arise when the titration of a residue is coupled to the global shift in the equilibrium population of the ensemble. Only a subset of all ionizable residues (E10, D19, D21, E75, D77, D83, D95, E101, E129, and E135) show high GCP values. These residues are in folded segments in the majority of the highly probable states under native conditions, are predicted to govern the energetics of the acidunfolding reaction, and are responsible for the cooperative character of this transition. $(B)$ The effects of point mutations on the stability of SNase at $\mathrm{pH} 7$ were measured by $\mathrm{GdnHCl}$-induced unfolding (25). $\Delta \Delta G_{\mathrm{pH}}$ describes the difference in stability between the WT protein and the Glu $\rightarrow$ Ala or Asp $\rightarrow$ Ala mutants. Also included in this plot are the four $\mathrm{His} \rightarrow$ Ala point mutants. $\mathrm{pH}_{\text {mid }}$ refers to the $\mathrm{pH}$ at the midpoint of the acid-induced unfolding, monitored by intrinsic fluorescence (25). The black curve describes the expected dependence of $\mathrm{pH}_{\text {mid }}$ on global stability, calculated as $\Delta G(\mathrm{pH})=\int \Delta \nu(\mathrm{pH})_{\mathrm{WT}} \partial \mathrm{pH}$. For variants that fall on or close to the curve (blue), the substituted amino acid is not coupled to the global unfolding transition. For variants that do not fall on this line (red), titration at the substituted site is coupled to the global structural transition. Comparison of $A$ and $B$ indicates that the substitutions that fall away from the curve are also the ones that are calculated to contribute to $\Delta Z$ in the WT ensemble (see Fig. $4 B$ ) and thus determine the $\mathrm{pH}$-dependent energetics of SNase.

tions in modulating the proton-linked global structural transition in SNase.

\section{Conclusions}

Two important implications about the character of local fluctuations and their impact on protein stability and function emerge from these studies. First, the results are consistent with the notion that the native-state ensemble of a protein is populated primarily by states that display a dual character, with some regions being compact and thermodynamically native-like and other regions being highly solvated and displaying the thermodynamic signature of unfolded-like regions (Fig. 1A). Interestingly, this dual character also has been observed in recent NMR spectroscopy studies that used cold denaturation to characterize structural features of the nativestate ensemble (59). In fact, the ensemble calculations described here qualitatively reproduced both the structural and thermodynamic features of the cold denaturation process (59). The ability of this simple ensemble model to reproduce such seemingly disparate processes as ligand-linked conformational equilibria, hydrogen exchange, and cold denaturation suggests that these different processes, and presumably many other solution processes of proteins, are governed by properties of a common set of structural/thermodynamic building blocks.

Second, these results explain mechanistically the manner in which conformational fluctuations can affect the apparent coupling between ligand binding and global structural transitions. Wyman's theory of linked functions (60) explains rigorously and quantitatively how the preferential binding of ligands can affect the equilibrium between two macroscopic conformational states of a protein that bind ligands with different affinities (e.g., the $\mathrm{T}$ and $\mathrm{R}$ states of hemoglobin). Linkage theory, however, does not contribute mechanistic insight about how this coupling is established, nor about the role of all but the most large-scale conformational changes. The ensemble-based analyses described here demonstrate that the effects of ligands on the conformational equilibrium between two macroscopic thermodynamic states (i.e., the native and acid unfolded states; see Fig. $3 A$ ) also can be exerted by differences in binding properties between microscopic elements of the same macroscopic state. In effect, by regulating the binding properties within the macroscopic native state, the stability difference between the native and denatured states can be effectively modulated.

This result has particularly important implications for the design and evolution of proteins. It suggests that the ligandsensitive equilibria of proteins can be fine-tuned simply by evolution of site-specific local stabilities within the protein. In the context described here, the evolution of the $\mathrm{pH}$ sensitivity of a macromolecule need not rely solely on the evolution of Coulomb, polar, or hydration interactions to modulate $\mathrm{pK}_{\mathrm{a}}$ values. Apparently, pH sensitivity also can be achieved by tuning the local stability of the microenvironments surrounding ionizable residues, which is equivalent to tuning the dielectric response of the protein (ref. 61 and C. A. Fitch, S.T.W., V.J.H., and B.G.-M.E., unpublished results). In a more general sense, the evolution of allosterism and of cooperativity entails the evolution of a precise balance between the energetics of local fluctuations, ligand-binding affinities, and global stability (62).

Finally, we note that the parameterized energy function used in these ensemble-based calculations $(41,43,47-55)$ is extremely simplified and provides only a coarse representation of the energy landscape accessible to each macroscopic state. It might seem paradoxical that such a simple representation of the energies of microstates could provide a unified phenomenological description of seemingly disparate equilibrium processes of proteins $(43-46,59,63)$. We interpret the success of this simple approach as evidence of the following: $(i)$ the observed properties of a protein are a manifestation of the 
energetic hierarchy of states in the ensemble, and (ii) that this hierarchy is a robust feature of each ensemble (i.e., not particularly sensitive to the precise features of the energy function) and therefore amenable to study with a coarse energetic description.

The success of the ensemble calculations suggests that many solution properties of proteins can be described quantitatively without having to go past the level of average properties of microstates and into the description of individual atoms and bonds. In this respect, the ensemble view of proteins constitutes a level of complexity intermediate between the unsophisticated treatment of proteins as strictly static structures and the daunting complexity of their treatment with explicit

1. Englander, S. W. (2000) Annu. Rev. Biophys. Biomol. Struct. 29, 213-238.

2. Bai, Y., Sosnick, T. R., Mayne, L. \& Englander, S. W. (1995) Science 269, 192-197.

3. Swint-Kruse, L. \& Robertson, A. D. (1996) Biochemistry 35, 171-180.

4. Hvidt, A. \& Nielsen, S. O. (1966) Adv. Protein Chem. 21, 287-386.

5. Chamberlain, A. K., Handel. T. \& Marqusee, S. (1996) Nat. Struct. Biol. 3, 782-787.

6. Radford, S. E., Buck, M., Topping, K. D., Dobson, C. M. \& Evans, P. A. (1992) Proteins 14, 237-248.

7. Fuentes, E. J. \& Wand, A. J. (1998) Biochemistry 37, 3687-3698.

8. Itzhaki, L. S., Neira, J. L. \& Fersht, A. R. (1997) J. Mol. Biol. 270, 89-98.

9. Lee, A. L., Kinnear, S. A. \& Wand, A. J. (2000) Nat. Struct. Biol. 7, 72-77.

10. Volkman, B. F., Lipson, D., Wemmer, D. E. \& Kern, D. (2001) Science 291, 2429-2433.

11. Yang, D. \& Kay, L. E. (1996) J. Mol. Biol. 263, 369-382.

12. Lu, H. P., Xun, L. \& Xie, X. S. (1998) Science 282, 1877-1882.

13. Yang, H., Luo, G., Karnchanaphanurach, P., Louie, T. M., Rech, I., Cova, S., Xun, L. \& Xie, X. S. (2003) Science 302, 262-266.

14. Honig, B. \& Nicholls, A. (1995) Science 268, 1144-1149.

15. Yang, A. \& Honig, B. (1994) J. Mol. Biol. 237, 602-614.

16. Yang, A. \& Honig, B. (1993) J. Mol. Biol. 231, 459-474.

17. Antosiewicz, J., McCammon, J. A. \& Gilson, M. K. (1994) J. Mol. Biol. 238, 415-436.

18. Bashford, D. \& Karplus, M. (1990) Biochemistry 29, 10219-10225.

19. Forsyth, W. R., Antosiewicz, J. M. \& Robertson, A. D. (2002) Proteins 48, $388-403$.

20. Edgcomb, S. P. \& Murphy, K. P. (2002) Proteins 49, 1-6.

21. Lee, K. K., Fitch, C. A. \& García-Moreno E., B. (2002) Protein Sci. 11, 1004-1016.

22. Tanford, C. (1970) Adv. Protein Chem. 24, 1-95.

23. Huang, Y. \& Bolen, D. W. (1995) Methods Enzymol. 259, 19-43.

24. Whitten, S. T. \& García-Moreno E., B. (2000) Biochemistry 39, 14292-14304

25. Whitten, S. T. (1999) Ph.D. dissertation (Johns Hopkins Univ., Baltimore).

26. García-Moreno E., B., Dwyer, J. J., Gittis, A. G., Spencer, D. S. \& Stites, W. E. (1997) Biophys. Chem. 64, 211-224.

27. Lee, K. K., Fitch, C. A., Lecomte, J. T. \& García-Moreno E., B. (2002) Biochemistry 41, 5656-5667.

28. Ackers, G. K. (1998) Adv. Protein Chem. 51, 185-253.

29. Ren, J., Kachel, K., Kim, H., Malenbaum, S. E., Collier, J. R. \& London, E. (1999) Science 284, 955-957.

30. Hogle, J. M. (2002) Annu. Rev. Microbiol. 56, 677-702.

31. Bullough, P. A., Hughson, F. M., Skehel, J. J. \& Wiley, D. C. (1994) Nature 371, 37-43.

32. Baker, D. \& Agard, D. A. (1994) Structure (London) 2, 907-910.

33. Gamblin, S. L., Haire, L. F., Russell, R. J., Stevens, D. J., Xiao, B., Ha, Y., Vasisht, N., Steinhauer, D. A., Daniels, R. S., Elliot, A., et al. (2004) Science 303, 1838-1842.

34. Ehrlich, L. S., Tianbo, L., Scarlatta, S., Chu, B. \& Carter, C. A. (2001) Biophys. J. 81, 586-594. "all-atom" representations of each accessible conformation. Indeed, an especially appealing feature of the ensemble-based approach described in this study is that it offers a computationally tractable method of apparently sufficient resolution to study and unify disparate processes $(46,63)$, reconcile the role of conformational fluctuations in modulating functionally important processes (64), and understand proteins in terms of their elementary building blocks (59).

This work was supported by National Institutes of Health Grant R01GM13747 (to V.J.H.), National Science Foundation Grants MCB9875689 (to V.J.H.) and MCB-0212414 (to B.G.-M.E.), and Welch Foundation Grant H-1461 (to V.J.H.).

35. Brooks, B. R. \& Karplus, M. (1983) Proc. Natl. Acad. Sci. USA 80, 6571-6575 36. Lazaridis, T. \& Karplus, M. (1999) Proteins 35, 133-152.

37. Rod, T. H., Radkiewicz, J. L. \& Brooks, C. L., III (2003) Proc. Natl. Acad. Sci. USA 100, 6980-6985.

38. McCammon, J. A. \& Harvey, S. (1987) Dynamics of Proteins and Nucleic Acids (Cambridge Univ. Press, Cambridge, U.K.).

39. Brooks, C. L., III, Karplus, M. \& Pettitt, B. M. (1988) Proteins: A Theoretical Perspective of Dynamics, Structure, and Thermodynamics, Advances in Chemical Physics (Wiley, New York).

40. Benkovic, S. J. \& Hammes-Schiffer, S. (2003) Science 301, 1196-1202.

41. Hilser, V. J. \& Freire, E. (1996) J. Mol. Biol. 262, 756-772.

42. Hynes, T. R. \& Fox, R. O. (1991) Proteins 10, 92-105.

43. Hilser, V. J. (2001) Methods Mol. Biol. 168, 93-116.

44. Hilser, V. J. \& Freire, E. (1997) Proteins Struct. Funct. Genet. 27, 171-183.

45. Hilser, V. J., Townsend, B. D. \& Freire, E. (1997) Biophys. Chem. 64, 69-79.

46. Hilser, V. J., Dowdy, D., Oas, T. G. \& Freire, E. (1998) Proc. Natl. Acad. Sci. USA 95, 9903-9908.

47. Murphy, K. P. \& Freire, E. (1992) Adv. Protein Chem. 43, 313-361.

48. Murphy, K. P., Bhakuni, V., Xie, D. \& Freire, E. (1992) J. Mol. Biol. 227, 293-306.

49. D‘Aquino, J. A., Gómez, J., Hilser, V. J., Lee, K. H., Amzel, L. M. \& Freire, E. (1996) Proteins Struct. Funct. Genet. 25, 143-156.

50. Gómez, J., Hilser, V. J., Xie, D. \& Freire, E. (1995) Proteins Struct. Funct. Genet. 22, 404-412.

51. Xie, D. \& Freire, E. (1994) J. Mol. Biol. 242, 62-80.

52. Baldwin, R. L. (1986) Proc. Natl. Acad. Sci. USA 83, 8069-8072.

53. Lee, K. H., Xie, D., Freire, E. \& Amzel, L. M. (1994) Proteins Struct. Funct. Genet. 20, 68-84.

54. Hebermann, S. M. \& Murphy, K. P. (1996) Protein Sci. 5, 1229-1239.

55. Luque, I., Mayorga, O. L. \& Freire, E. (1996) Biochemistry 35, 13681-13688.

56. Matthew, J. B., Gurd, F. R., García-Moreno E., B., Flanagan, M. A., March, K. L. \& Shire, S. J. (1985) CRC Crit. Rev. Biochem. 18, 91-197.

57. Schaefer, M., van Vlijmen, H. W. T. \& Karply, M. (1998) Adv. Protein Chem. 51, 1-57.

58. Fitch, C. A., Karp, D. A., Lee, K. K., Stites, W. E., Lattman, E. E. \& Garcia-Moreno E., B. (2002) Biophys. J. 82, 3289-3304.

59. Babu, C. R., Hilser, V. J. \& Wand, A. J. (2004) Nat. Struct. Biol. 11, 352-357.

60. Wyman, J. (1964) Adv. Protein Chem. 19, 223-286.

61. Schutz, C. N. \& Warshel, A. (2001) Proteins 44, 400-417.

62. Luque, I., Leavitt, S. A. \& Freire, E. (2002) Annu. Rev. Biophys. Biomol. Struct. 31, 235-256.

63. Pan, H., Lee, J. C. \& Hilser, V. J. (2000) Proc. Natl. Acad. Sci. USA 97, 12020-12025.

64. Ferreon, J. C., Volk, D. E., Luxon, B. A., Gorenstein, D. G. \& Hilser, V. J. (2003) Biochemistry 42, 5582-5591.

65. DeLano, W. L. (2002) The PYMOL Molecular Graphics System (DeLano Scientific, San Carlos, CA). 